\title{
Management of the respiratory distress symptom cluster in lung cancer: a randomised controlled feasibility trial
}

\author{
Janelle Yorke $^{1,2}$ - Mari Lloyd-Williams ${ }^{3}$. Jacky Smith ${ }^{4,5}$. Fiona Blackhall ${ }^{2,5}$. \\ Amelie Harle $^{2}$ • June Warden ${ }^{1}$ • Jackie Ellis ${ }^{3}$ • Mark Pilling ${ }^{1} \cdot$ Jemma Haines $^{6}$. \\ Karen Luker $^{1} \cdot$ Alex Molassiotis ${ }^{1,7}$
}

Received: 24 March 2015 / Accepted: 8 June 2015 / Published online: 26 June 2015

(C) The Author(s) 2015. This article is published with open access at Springerlink.com

\begin{abstract}
Background Breathlessness, cough and fatigue are distressing symptoms for patients with lung cancer. There is evidence that these three symptoms form a discreet symptom cluster. This study aimed to feasibly test a new non-pharmacological intervention for the management of the Respiratory Distress Symptom Cluster (breathlessness-cough-fatigue) in lung cancer.

Method This was a multi-centre, randomised controlled nonblinded parallel group feasibility trial. Eligible patients (patients with primary lung cancer and 'bothered' by at least two of the three cluster symptoms) received usual care plus a multicomponent intervention delivered over two intervention training sessions and a follow-up telephone call or usual care only. Follow-up was for 12 weeks, and end-points included six numerical rating scales for breathlessness severity,
\end{abstract}

Trial registration: RCT registration at Current Controlled Trials ISRCT N13173844.

Alex Molassiotis

alex.molasiotis@polyu.edu.hk

1 School of Nursing, Midwifery and Social Work, University of Manchester, Manchester, UK

2 The Christie NHS Foundation Trust, Manchester, UK

3 Health Services Research, University of Liverpool, Liverpool, UK

4 Centre for Respiratory and Allergy, University Hospital South Manchester, Manchester, UK

5 Faculty of Medicine and Human Sciences, University of Manchester, Manchester, UK

6 Airways Clinic Services, Lancashire Teaching Hospitals Foundation Trust, Lancashire, UK

7 School of Nursing, The Hong Kong Polytechnic University, Hung Hom, Hong Kong, China
Dyspnoea-12, Manchester Cough in Lung Cancer scale, FACIT-Fatigue scale, Hospital Anxiety and Depression scale, Lung Cancer Symptom Scale and the EQ-5D-3L, collected at baseline, week 4 and week 12 .

Results One hundred seven patients were randomised over 8 months; however, six were removed from further analysis due to protocol violations (intervention group $n=50$ and control group $n=51)$. Of the ineligible patients $(n=608), 29 \%$ reported either not experiencing two or more symptoms or not being 'bothered' by at least two symptoms. There was $29 \%$ drop-out by week 4 , and by week 12 , a further two patients in the control group were lost to follow-up. A sample size calculation indicated that 122 patients per arm would be needed to detect a clinically important difference in the main outcome for breathlessness, cough and fatigue.

Conclusions The study has provided evidence of the feasibility and acceptability of a new intervention in the lung cancer population and warrants a fully powered trial before we reach any conclusions. The follow-on trial will test the hypothesis that the intervention improves symptom cluster of breathlessness, cough and fatigue better than usual care alone. Full economic evaluation will be conducted in the main trial.

Keywords Lung cancer - Non-pharmacological intervention · Self-management · Symptoms · Symptom cluster .

Dyspnoea $\cdot$ Breathlessness $\cdot$ Cough $\cdot$ Fatigue $\cdot$ Respiratory symptoms

\section{Introduction}

Breathlessness, cough and fatigue are distressing symptoms for patients with lung cancer [1,2]. Breathlessness occurs in up to $90 \%$ of patients and is distressing for both patients and their carers, with a deleterious effect on health related quality of life 
[3]. Cough occurs in $47-86 \%$ of patients, and for some, it can be more distressing than breathlessness [4]. Patients report fatigue (prevalence 50-90 \%) as one of the most important and distressing symptoms related to cancer and its treatment [5]. Patients often experience multiple symptoms. Longitudinal data suggests that breathlessness, cough and fatigue form a discrete cluster, named the Respiratory Distress Symptom Cluster [1]; patients with one symptom will often suffer from one or both of the others [6]. This has implications for management, as potentially, the symptoms in the cluster need to be managed concurrently if we are to observe significant improvements in individual symptoms that are part of the cluster.

Despite the complexity of symptomatology in people with lung cancer, non-pharmacological symptom management intervention research has focused on breathlessness, cough and fatigue as single symptoms [7]. Breathlessness selfmanagement techniques are clinically cost-effective [8-10]. Interventions such as exercise and acupressure are effective in the management of cancer-related fatigue [11-13], but these have not been robustly tested in intrathoracic malignancies. Cough has not received the same attention as other cancer symptoms, although there is a developing evidence-base to support non-drug measures for cough $[14,15]$. However, since these symptoms often occur together, it is important to rigorously test interventions that target multiple symptoms. To address this gap, we applied the Medical Research Council (MRC) framework for the development and evaluation of complex interventions [16] to develop an intervention for the (self) management of the respiratory distress symptom cluster (breathlessnesscough-fatigue). The work conducted during the intervention theory and modelling development stages has been previously reported [17-19] and centres around the views of patients, their caregivers and health professionals on the components of a non-pharmacological intervention for the management of the symptom cluster as well as preferences of delivery of the intervention.

This paper reports the findings of a feasibility randomised trial of a new intervention, the Respiratory Distress Symptom Intervention (RDSI). The main objectives were to

1. Assess the feasibility and acceptability of the intervention to patients

2. Test the design and practicality of the protocol with regard to recruitment, patient withdrawal and completion of assessments

3. Examine the performance of the different symptom outcome measures in order to inform a power calculation for the follow-on trial

4. Provide proof of principle for any positive contribution of the intervention to the management of the symptom cluster

\section{Methods}

\section{Study design}

This study was a non-blinded randomised feasibility trial comprising two groups: (i) intervention group receiving usual care and RDSI and (ii) control group receiving usual care only. Both groups also received the Macmillan breathlessness and fatigue information booklets.

\section{Participants and settings}

This multisite study had 11 participating centres, including seven secondary care teaching hospitals, two specialist cancer centres and two district general hospitals, located in the north of England. Patients from each of the participating sites attending lung cancer out-patient clinics were screened for eligibility by the research nurse in collaboration with the attending clinician. Eligible participants were adults with primary lung cancer, an expected prognosis of at least 3 months, a World Health Organisation (WHO) Performance Status 0-2, and reported being 'bothered' from at least two of the cluster symptoms: breathlessness or cough or fatigue, in any combination. Patients were screened for symptom eligibility by asking (i) Do you have breathlessness/cough/fatigue, and if 'yes', (ii) Are you bothered by breathlessness/cough/fatigue? Patients were excluded if they had an acute exacerbation of chronic obstructive pulmonary disease (COPD) or chest infection within the past 4 weeks that necessitated a change in medication, received radiotherapy to the chest or chemotherapy in the previous 4 weeks, or had surgical treatment for lung cancer in the previous 6 weeks.

\section{The intervention}

RDSI includes core components that were most highly rated by patients, carers and clinicians in our previous work [17-19] and with the strongest evidence-base:

1. Controlled breathing techniques: consisting of diaphragmatic breathing exercises and calming techniques practiced twice a day and used as needed for episodes of intense breathlessness and/or anxiety [8-10].

2. Cough easing techniques: includes education (capacity for voluntary cough easing), identifying warning signs for cough and using modified swallow technique/ relaxed throat breathing $[14,15]$.

3. Acupressure: a small number of acupressure points are taught: L7, L9, LI4 (located on the hand and wrist areas), CV21 and 22 (sternum), and ST36 (knee). Patients apply pressure for $1 \mathrm{~min}$ at least twice a day [11-13]. The selection of points and the approach to acupressure selected was based on reviewing the literature and obtaining 
consensus with acupuncture-trained therapists. Points were selected to affect all three symptoms of the cluster, some based on our previous work too.

4. Information pack: supplementary written information giving practical advice about symptom experience and communication strategies, sleep hygiene, activity management/ energy conservation strategies, anxiety management techniques (such as the 'calming hand' technique) and carer support, all collated from existing resources.

Training for all components included psycho-educational counselling for internalising locus of control, acceptance that learning the techniques is hard work and requires frequent practice and setting realistic goals. Patients were taught the core components individually during an initial face-to-face session lasting up to $1 \mathrm{~h}$ and then repeated 1 week later together with discussion on the supplementary information. The information pack was given to the patient on the first intervention delivery session and discussed at the second visit. Patients also received one follow-up telephone call 2 weeks after the second face-to-face training session. The intervention was delivered in a community setting (in the patient's home or in a hospital/community facility near to a patient's home). The intervention was delivered by specialist nurses, physiotherapists and complementary therapists who received specific training from the complementary therapy team at The Christie Hospital NHS Trust, to deliver the intervention. Training consisted of one face-to-face group session lasting $3 \mathrm{~h}$ and a follow-up refresher session half way through the trial. To collect self-report details regarding use and perceptions of usefulness of the different intervention techniques, patients in the treatment group were requested to complete a daily diary for the first 4 weeks, then weekly until week 12 . To assess how 'useful' each core component was, participants were asked to tick one of the following: scaled as 'not at all', 'a little bit', 'quite a bit', and 'very much'.

\section{Outcome assessments}

As this was a feasibility study, there were no designated primary or secondary outcome measures. Outcome assessments for both patients and carers were collected at (i) baseline (before randomisation), (ii) week 4 (end of follow-up contact in RDSI group) and (iii) week 12 (end of study). Participants allocated to the intervention group were asked to complete a daily diary for the first 4 weeks then weekly until week 12 . The diary was used to collect process data such as frequency of use of the different core intervention components and their perceived usefulness.

Breathlessness was assessed using six $0-10$ numerical rating scales (NRS) including average and worst breathlessness in the past $24 \mathrm{~h}$, distress and unpleasantness associated with breathlessness (higher scores worse), and relief from breathlessness and ability to cope with breathlessness (higher scores better) $[10,20]$. Breathlessness was assessed using the Dyspnoea-12 (D-12) scale, a measure which consists of 12 items that measure a patient's current level of breathlessness severity incorporating physical and emotional domains (scores range from 0 to 36, higher scores worse breathlessness) [21-23]. Its Cronbach alpha for the current study population was excellent $(\alpha=0.9)$. Cough was assessed using the Manchester Cough in Lung Cancer scale (MCLC), a 10-item self-report questionnaire (scores range from 1 to 50, higher scores worse) [24]. Fatigue was assessed using the 13-item Functional Assessment of Cancer Therapy-Fatigue (FACITF) (scores range from 0 to 52, higher scores worse) [25].

Non-single symptom questionnaires included the Lung Cancer Symptom Scale, a nine-item visual analogue scale used to assess lung cancer-related symptoms (scores range from 0 to $100 \mathrm{~mm}$ for each of the nine items, higher score denotes more severe symptom experience) [26], the EQ-5D-3L (index and visual analogue scale (VAS)) was used to describe and value overall perceived health-related quality-of-life and the feasibility of collecting a preference-based measure of health status for use in an economic evaluation within the follow-on trial [27], the EQ-5D-3 was valued using UK population norms [28], and the Hospital Anxiety and Depression scale (HADS) [29] was used to measure anxiety and depression. The HADS consists of a separate scale for anxiety and depression, each consisting of seven items with scores ranging from 0 to 21 (higher scores denote poorer outcome).

For each self-reported outcome measure, patients were requested to rate on a NRS 0 to 10 scale its 'ease of use' (higher score denotes greater ease of use) and 'relevance' to their experience (higher score denotes greater relevance).

\section{Sample and randomisation}

As this was a feasibility study, no sample size calculation was performed, and the convention of 30 patients per arm at the end of the study (i.e. week 12) was used [30]. To achieve this, the aim was to randomise 120 patients (60 patients per trial arm) with an additional 60 carers, as available, to accommodate an anticipated high attrition of $50 \%$ to last assessment. The randomisation method consisted of minimisation (with a random element) controlling for gender, age (69 or less; 70 and above) and lung cancer treatment group, and was conducted by the Clinical Trial Unit at The Christie Hospital, Manchester. The lung cancer treatment group included (i) no further cancer therapy for patients who are being treated symptomatically only, (ii) post-curative treatment for patients who have completed potentially curative cancer therapy who are on follow-up (i.e. no further cancer therapy is planned unless they relapse) and (iii) on palliative cancer therapy follow-up for patients who have incurable cancer. 


\section{Ethical approval}

Approval for the conduct of this study was provided by the National Health Service (NHS) ethics and participating hospital governance committees (NHS reference: 12/ NW/0090). All participants provided informed signed consent.

\section{Statistical methods}

The SPSS (v20) statistical computer package was used for analysis. Generalised estimating equation (GEE) models were applied to test the group effect, time effect and their interaction. GEE automatically handle missing data via correlation matrices and are generally robust to the choice of correlation structure $[31,32]$. The estimated marginal means and their pairwise comparison $p$ values for all scales by group and time point were calculated, and basic $t$ tests were also considered as a sensitivity analysis to check the conclusions from these models.

Minimal clinically important differences (MCID) for the key symptom outcome measures were calculated using two main methods: anchor-based and distributionbased methods [33-35] and were used to calculate the sample size required for the follow-on trial. For distribution-based calculations, the effect size (change after intervention divided by standard deviation of baseline scores) was used to determine the lowest meaningful detectable difference based on the underlying variability of the reported data [35]. A medium effect size of 0.5 was used. We applied this distribution-based method for each main outcome for each symptom: Dyspnoea-12, MCLCS and the FACIT-F. Anchor-based methods can use regression and a known MCID for a related variable or can use a patient-rated impression of global change for use in a receiver operator characteristic (ROC) cut-point analysis to provide an anchor to define a small, medium or large change [35]. Specifically, a reduction (week12 baseline) in NRS worst breathlessness was used to define a worsening (status $=0$ ) and an increase as an improvement (status $=1$ ) for use in the ROC analysis. To calculate the anchor-based MCID for Dyspnoea-12, we used the previously reported NRS 'breathlessness severity' (i.e. regression between D-12 and NRS, then using the MCID for the NRS in the regression equation to gain the MCID for D-12) and for the FACIT-F previously reported MCID's for different patient populations [36,37]. No appropriate anchor was available for the MCLC. There are recognised limitations for both methods of MCID calculation, and therefore, it is recommended that both MCID methods are applied, where possible, to frame the categorisation of effect size in the context of patient report [35].

\section{Results}

\section{Feasibility aspects}

\section{Participants}

Figure 1 (CONSORT diagram) illustrates that 715 patients were screened, and 107 eligible patients were randomised between April 2013 and November 2013 to one of the two trial treatment arms (intervention group (RDSI) $n=$ 53 and control group $n=54)$. Of the ineligible patients $(n=608), 29 \%$ reported either not experiencing two or more symptoms or not being bothered by at least two symptoms. The average age of the patients was 67.7 years (SD 9.6 years); $54(53 \%)$ were female and most $(60 \%)$ reported all three symptoms at baseline. The two groups were different in baseline COPD prevalence (control group $50 \%$ and RDSI group $28 \%$ ) and Lung Cancer Symptom Scale (higher mean scores in the control group (421) compared to the RDSI group (352) (Table 1).

\section{Data completeness}

Of the 107 randomised patients, four were found to not meet the inclusion criteria $(\operatorname{RDSI}=2$ and control $=2$ ) and were removed from data analysis. A further two did not provide any data at baseline; therefore, they were removed from the analysis since they had not strictly followed protocol. The total sample analysed was 101 patients (RDSI $n=50$ and control $n=51$ ).

By week 4 29/101 (29\%), patients were withdrawn, and by week 12, a further two patients in the control group were lost to follow-up. No patients in the RDSI group were lost between week 4 and week 12 . Total attrition at 12 weeks was 30/101 (29.7 \%). Since there was very little attrition after week 4 , we conducted sample size analyses based on observations at week 12.

At week 12, there were more drop-outs in the treatment group (38\%) than the control group (24\%); however, this was not statistically significant $\left(\chi^{2}=2.5, d f=1, p=0.1\right)$. Logistic regression for drop-out status using age, gender, treatment type and WHO score revealed no significant items. Individually, only the WHO score was borderline significantly associated with drop-out $\left(\chi^{2}=4.0, d f=1, p=0.044\right)$. GEE models with an unstructured correlation parameterisation were used, because of the differently spaced time intervals for assessments.

\section{Outcomes assessments}

The six NRS breathlessness scales combined scored the lowest for ease of use $(6.5 / 10)$ and relevance $(6.8 / 10)$, and 


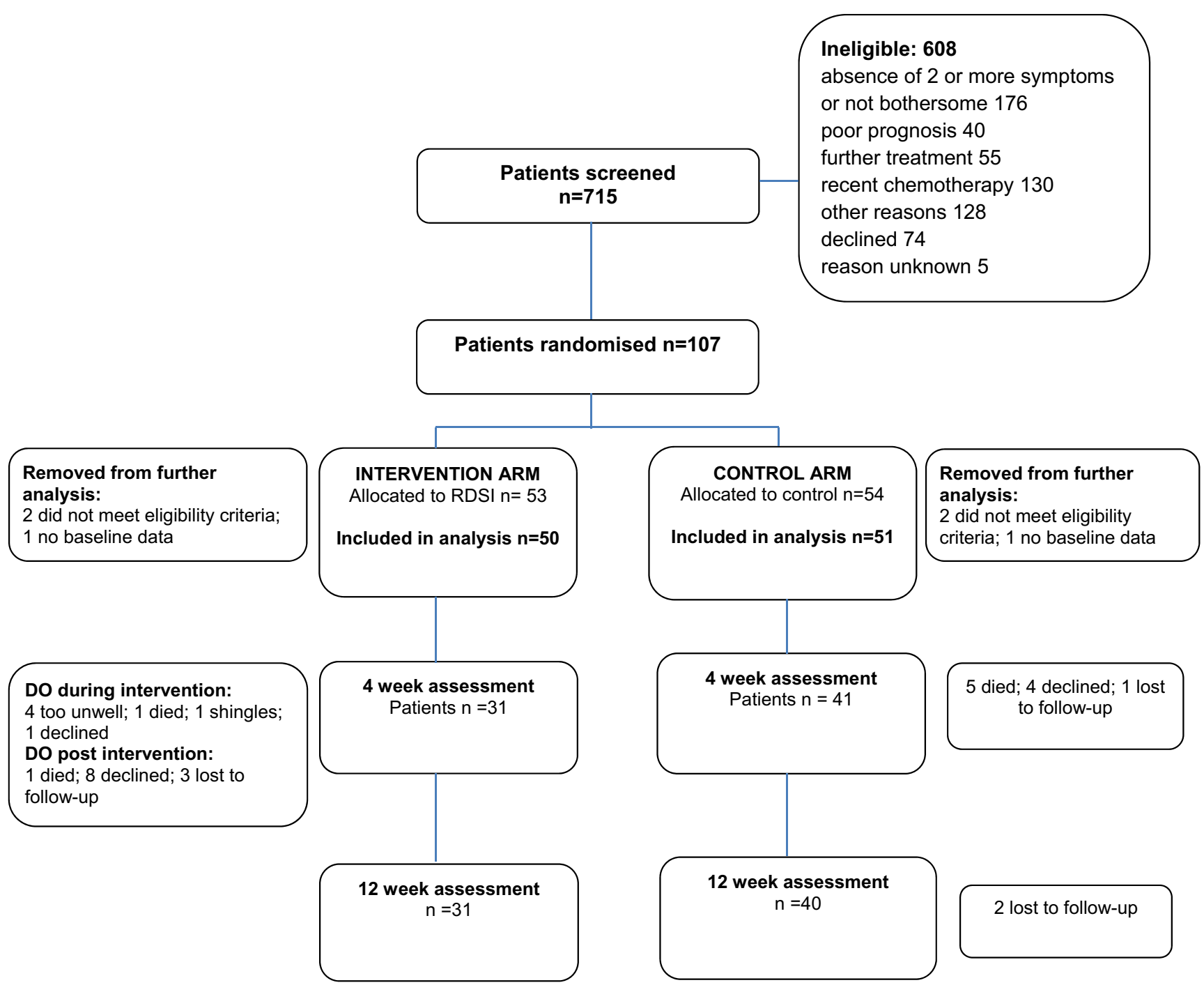

Fig. 1 Study flow chart. RDSI Respiratory Distress Symptom Intervention, DO drop-out

the EQ-5D-3L was the best for ease of use $(7.8 / 10)$ and relevance $(7.8 / 10)$ (Table 2).

\section{Respiratory symptom cluster outcomes}

Results for the respiratory symptom cluster outcomes (breathlessness, cough and fatigue measures) are presented as change in mean scores from baseline to 4 and 12 weeks (Table 3). Figure 2 demonstrates variability of the scores for each symptom outcome measure at baseline, week 4 and week 12, although with a trend in scores to show some improvement in the RDSI group. Dyspnoea-12 was chosen as the primary outcome for breathlessness for the follow-on trial because it was significant in the GEE model at week 12 for the RDSI group (see below), and patients rated it higher (scale of $0-10$, higher is better) than the set of NRS breathlessness scales for ease of completion (6.7 versus 6.5$)$ and relevance (7.1 versus 6.8) (Table 2).
Non-respiratory symptom cluster outcomes

The mean change from baseline to week 4 and baseline to week 12 for the Lung Cancer Symptom Scale, HADS and EQ-5D-3L is shown in Table 3, again with a positive pattern in terms of improvement in the RDSI group.

The GEE models included the WHO score as a covariate due to its potential relationship with drop-outs, although it was usually non-significant. In a number of models (consistent with Fig. 2), time was a significant term for some endpoints (NRS 'worst breathlessness' and NRS 'distress from breathlessness', Dyspnoea-12 and MCLC), and group was significant for others (Dyspnoea-12, EQ-5D-3L, Lung Cancer Symptom Scale) due to imbalances at baseline. However, when the differential effect of group was considered (i.e. the group $\times$ time interaction term), only EQ-5D-3L was significant ( $p=0.042$ respectively) and was due to a change at week 12 (marginal mean difference at week 12 of -0.17 (SD 0.06, $95 \%$ CI $[-0.04,-0.30]), p=0.009)$. The group differences at 
Table 1 Baseline sample characteristics

\begin{tabular}{|c|c|c|}
\hline Variable & Control $(n=51)$ & RDSI-LC $(n=50)$ \\
\hline Age (mean (SD)) & $67.6(9.1)$ & $67.8(10.1)$ \\
\hline \multicolumn{3}{|l|}{ Gender frequency $(\%)$} \\
\hline Male & $25(49.0)$ & $22(44.0)$ \\
\hline Female & $26(51.0)$ & $28(56.0)$ \\
\hline \multicolumn{3}{|l|}{ Symptom cluster frequency (\%) } \\
\hline \multicolumn{3}{|l|}{ Breathlessness } \\
\hline Present & $50(98.0)$ & $49(98.0)$ \\
\hline Bothersome (of those with breathlessness 'present') & $50(100.0)$ & $48(98.0)$ \\
\hline \multicolumn{3}{|l|}{ Cough } \\
\hline Present & $41(80.4)$ & $40(80.0)$ \\
\hline Bothersome (of those with cough 'present') & $32(78.0)$ & $35(83.3)$ \\
\hline \multicolumn{3}{|l|}{ Fatigue } \\
\hline Present & $51(100.0)$ & $48(96.0)$ \\
\hline Bothersome (of those with fatigue 'present') & $49(96.1)$ & $48(100.0)$ \\
\hline \multicolumn{3}{|l|}{ Number of symptoms frequency (\%) } \\
\hline 2 & $22(43.1)$ & $18(36.0)$ \\
\hline 3 & $29(56.9)$ & $32(64.0)$ \\
\hline \multicolumn{3}{|l|}{ WHO score frequency $(\%)$} \\
\hline Restricted in strenuous activity & $21(52.5)$ & $25(61.0)$ \\
\hline Ambulatory/capable of all self-care & $19(47.5)$ & $16(39.0)$ \\
\hline \multicolumn{3}{|l|}{ Patient treatment group frequency $(\%)$} \\
\hline No further active cancer therapy & $7(13.7)$ & $5(10.0)$ \\
\hline Post-curative treatment & $18(35.3)$ & $19(38.0)$ \\
\hline Palliative cancer therapy follow-up & $26(51.0)$ & $26(52.0)$ \\
\hline \multicolumn{3}{|l|}{ COPD frequency $(\%)$} \\
\hline Yes & $25(50.0)$ & $14(28.0)$ \\
\hline No & $25(50.0)$ & $36(72.0)$ \\
\hline \multicolumn{3}{|l|}{ Prescribed opioids } \\
\hline Yes & $18(36.7)$ & $16(32.0)$ \\
\hline \multicolumn{3}{|l|}{ Prescribed benzodiazepines } \\
\hline Yes & $2(4.0)$ & $3(6.0)$ \\
\hline
\end{tabular}

RDSI Respiratory Distress Symptom Intervention, COPD chronic obstructive pulmonary disease week 12 were also estimated, and only Dyspnoea-12 had a significant result (marginal mean 5.19, SD 2.33, 95\% CI [0.62, 9.75], Wald $\chi^{2}=1.27, d f=2, p=0.026$ ), where the reduction (improvement) was greatest in the RDSI group (Table 4).

\section{Sample size calculations for follow-on trial}

Attrition in the feasibility trial was $29.7 \%$ and was highest in the palliative care treatment group. The largest correlation of Dyspnoea-12 to other breathlessness questionnaires used in the feasibility study was the NRS worst breathlessness $(r=0.416)$ which has a MCID of 1 unit [20,33,35]. Using an anchor-based regression analysis, a $1 \mathrm{U}$ change in worst breathlessness was equivalent to a $1.22 \mathrm{U}$ change in Dyspnoea-12. Using the same anchor, an ROC analysis estimated a cut-point (maximising Youden's J) and resulted in a Dyspnoea-12 MCID of 2.5 U.
A distribution-based analysis using a baseline effect size of 0.5 produced a Dyspnoea-12 MCID of 4.7. Based on these analyses, we have taken a Dyspnoea-12 MCID conservative estimate of $3 \mathrm{U}$ to use in the sample size calculation. Since three different variables are used as co-primary endpoints, to maintain an overall significance of $5 \%$, the separate parts of the sample size are based on an alpha of $5 \% / 3$. To detect a difference of $3 \mathrm{U}$ in Dyspnoea-12, a sample size of 97 patients per arm, with $80 \%$ power, at $5 \% / 3$ significance (for a one-sided $t$ test at week 12, adjusted for large correlation to baseline values [37]). Allowing for $20 \%$ attrition at week 12, then 122 patients per arm should be recruited.

Using data from the sub-group of patients with 'bothersome' cough (66\% of the total sample), the MCLC had a mean difference between the two treatment groups of $3.0 \mathrm{U}$. A distribution-based analysis using an effect size of 0.5 produced 
Table 2 Patient preference at baseline for the different outcome measures

\begin{tabular}{|c|c|c|c|c|c|c|}
\hline & $N$ & Mean (SD) & $N$ & $\begin{array}{l}\text { Control } \\
\text { Mean (SD) }\end{array}$ & $N$ & $\begin{array}{l}\text { RDSI } \\
\text { Mean (SD) }\end{array}$ \\
\hline \multicolumn{7}{|l|}{ Six NRS combined } \\
\hline How easy to complete & 101 & $6.5(2.9)$ & 51 & $6.3(3)$ & 50 & $6.7(2.9)$ \\
\hline How relevant & 101 & $6.8(2.9)$ & 51 & $7.2(2.6)$ & 50 & $6.5(3.1)$ \\
\hline \multicolumn{7}{|l|}{ Dyspnoea-12 } \\
\hline How easy to complete & 101 & $6.6(2.9)$ & 51 & $6.3(3)$ & 50 & $6.8(2.8)$ \\
\hline How relevant & 101 & $7.1(2.8)$ & 51 & $7(2.8)$ & 50 & $7.1(2.8)$ \\
\hline \multicolumn{7}{|c|}{ Manchester Cough in Lung Cancer Scale } \\
\hline How easy to complete & 91 & $7.3(2.7)$ & 47 & $7.2(2.8)$ & 44 & $7.4(2.5)$ \\
\hline How relevant & 91 & $7.6(2.4)$ & 47 & $7.7(2.3)$ & 44 & $7.5(2.5)$ \\
\hline \multicolumn{7}{|l|}{ Fatigue (FACIT-F) } \\
\hline How easy to complete & 100 & $7.3(2.6)$ & 51 & $6.9(2.6)$ & 49 & $7.7(2.6)$ \\
\hline How relevant & 100 & $7.8(2.4)$ & 51 & $7.8(2.3)$ & 49 & $7.9(2.5)$ \\
\hline \multicolumn{7}{|c|}{ Hospital anxiety and depression scale } \\
\hline How easy to complete & 99 & $7.5(2.4)$ & 51 & $7.3(2.5)$ & 48 & $7.7(2.5)$ \\
\hline How relevant & 99 & $7.5(2.6)$ & 51 & $7.3(2.8)$ & 48 & $7.8(2.2)$ \\
\hline \multicolumn{7}{|c|}{ Lung Cancer Symptom Scale } \\
\hline How easy to complete & 98 & $7.3(2.6)$ & 50 & $6.7(3.1)$ & 48 & $8.1(1.6)$ \\
\hline How relevant & 98 & $7.6(2.5)$ & 50 & $7.3(2.9)$ & 48 & $7.8(1.9)$ \\
\hline \multicolumn{7}{|l|}{ EQ-5D } \\
\hline How easy to complete & 101 & $7.8(2.5)$ & 51 & $7.4(2.7)$ & 50 & $8.2(2.2)$ \\
\hline How relevant & 101 & $7.8(2.7)$ & 51 & $7.8(2.5)$ & 50 & $7.7(2.8)$ \\
\hline
\end{tabular}

NRS numerical rating scale, EQ-5D Euro-Qual, $R D S I$ Respiratory Distress Symptom Intervention

Table 3 Analysis of respiratory symptom cluster outcomes: mean change scores from baseline to week 4 and baseline to week 12

\begin{tabular}{|c|c|c|c|c|}
\hline & \multicolumn{2}{|c|}{ Mean (SD) difference (week 4-baseline) } & \multicolumn{2}{|c|}{ Mean (SD) difference (week 12-baseline) } \\
\hline & Control & RDSI & Control & RDSI \\
\hline \multicolumn{5}{|l|}{ Breathlessness-NRS } \\
\hline Average breathlessness & $-0.38(2.21)$ & $-0.06(1.55)$ & $-0.75(2.33)$ & $-0.17(2.00)$ \\
\hline Worst breathlessness & $-0.38(2.55)$ & $-0.45(2.06)$ & $-0.56(2.7)$ & $-0.34(2.59)$ \\
\hline Relief breathlessness & $-0.55(3.27)$ & $0.41(3.16)$ & $-0.38(3.34)$ & $0.68(3.5)$ \\
\hline Distress breathlessness & $0.03(2.26)$ & $-0.63(1.9)$ & $-0.88(2.9)$ & $-1.07(2.07)$ \\
\hline Ability to cope & $0.81(2.74)$ & $0.5(2.62)$ & $0.5(2.97)$ & $1.03(2.63)$ \\
\hline Unpleasant & $0.13(2.97)$ & $-0.27(2.23)$ & $-0.25(3.09)$ & $-0.07(2.39)$ \\
\hline Dyspnoea-12 & $-2.13(7.41)$ & $-2.98(7.46)$ & $-1.52(8.31)$ & $-3.04(7.78)$ \\
\hline Cough-MCLC & $-1.00(6.33)$ & $-1.69(5.91)$ & $-2.26(8.84)$ & $-0.86(7.02)$ \\
\hline Fatigue-FACIT & $-2.44(9.19)$ & $-1.45(9.5)$ & $-1.28(9.47)$ & $-1.6410 .01)$ \\
\hline Lung Cancer Symptom Scale & $-17.83(132.79)$ & $-27.68(129.54)$ & $-21.90(121.71)$ & $7.48(118.88)$ \\
\hline \multicolumn{5}{|l|}{ HADS } \\
\hline Anxiety scale & $0.06(2.85)$ & $0.31(3.08)$ & $-0.06(3.10)$ & $0.81(2.75)$ \\
\hline Depression scale & $0.58(2.66)$ & $0.64(2.39)$ & $1.22(3.97)$ & $0.70(3.14)$ \\
\hline EQ-5D Score & $0.02(0.29)$ & $0.06(0.23)$ & $-0.05(0.33)$ & $0.06(0.28)$ \\
\hline EQ-5D Health State Today (VAS) & $-3.30(18.94)$ & $0.40(24.14)$ & $-4.50(21.03)$ & $-2.20(2.53)$ \\
\hline
\end{tabular}

NRS numerical rating scale, MCLC Manchester Cough in Lung Cancer Scale, HADS Hospital Anxiety and Depression Scale, EQ-5D Euro-Qual, VAS visual analogue scale, RDSI Respiratory Distress Symptom Intervention 

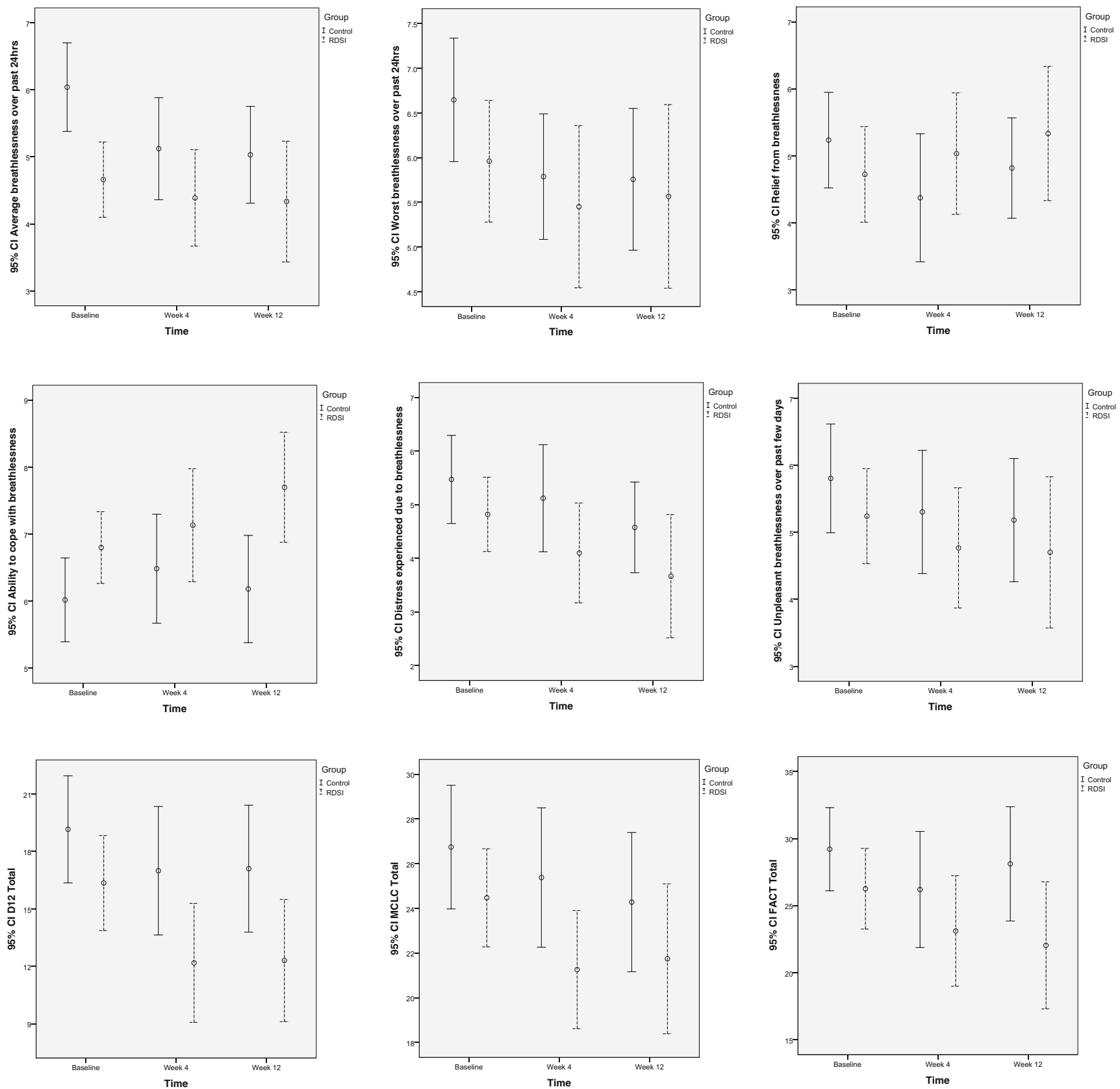

Fig. 2 Mean scores $(95 \% \mathrm{CI})$ from baseline to 4 and 12 weeks. RDSI Respiratory Distress Symptom Intervention, D12 Dyspnoea-12 questionnaire, $M C L C$ Manchester Cough in Lung Cancer questionnaire, FACT-F Functional Assessment of Chronic Illness Therapy-Fatigue

a MCLC MCID of 4.4. Based on these analyses, a MCLC MCID estimate of $3 \mathrm{U}$ was used in the sample size calculation. To detect a difference of $3 \mathrm{U}$ in MCLC, a sample size of 81 patients per arm at week 12 is required, and allowing for attrition at week 12, then 102 per am should be recruited.

For fatigue, the FACIT-F was used which has a published MCID of 3 to 4 U [36,38]. The FACIT-F had a mean difference between the two treatment groups of $4.8 \mathrm{U}$. Therefore, a FACIT-F MCID of $4 \mathrm{U}$ was used in the sample size calculation. To detect a difference of $4 \mathrm{U}$ in FACIT-F, a sample size of 80 patients per arm at week 12 is required, and allowing for attrition at week 12, then 100 per arm should be recruited. Therefore, by recruiting to the higher number required (i.e. 122 patients per arm) will provide $80 \%$ power that meaningful differences could be detected in all three symptoms in the cluster, with overall type I error rate of $5 \%$.

\section{Adherence to and perceptions of usefulness of the intervention components}

Between 19 and 32 patients completed the daily and weekly diaries at any time point. Participants reported high adherence 
Table 4 Analysis of respiratory symptom cluster outcomes: GEE differences between groups, with 95\%CI

\begin{tabular}{|c|c|c|c|c|c|c|}
\hline & \multicolumn{3}{|c|}{ Mean (SD) group difference at week 4} & \multicolumn{3}{|c|}{ Mean (SD) group difference at week 12} \\
\hline & Control-RDSI & $95 \% \mathrm{CI}$ & $p$ value & Control-RDSI & $95 \% \mathrm{CI}$ & $p$ value \\
\hline \multicolumn{7}{|l|}{ Breathlessness-NRS } \\
\hline Average breathlessness & $0.64(0.50)$ & $-0.34,1.61$ & 0.2 & $0.65(0.58)$ & $-0.49,1.80$ & 0.26 \\
\hline Worst breathlessness & $0.45(0.55)$ & $-0.63,1.53$ & 0.42 & $0.41(0.65)$ & $-0.86,1.67$ & 0.53 \\
\hline Relief breathlessness & $-0.58(0.71)$ & $-1.97,0.81$ & 0.42 & $-0.30(0.66)$ & $-1.60,1.00$ & 0.65 \\
\hline Distress breathlessness & $1.46(0.63)$ & $0.23,2.69$ & 0.02 & $0.75(0.70)$ & $-0.63,2.13$ & 0.29 \\
\hline Ability to cope & $-1.02(0.63)$ & $-2.24,0.21$ & 0.1 & $-1.01(0.62)$ & $-2.22,0.21$ & 0.11 \\
\hline Unpleasant & $0.71(0.63)$ & $-0.52,1.94$ & 0.26 & $0.52(0.75)$ & $0.94,1.99$ & 0.49 \\
\hline \multicolumn{7}{|l|}{ Dyspnoea-12 } \\
\hline Total & $4.32(2.22)$ & $-0.03,8.67$ & 0.05 & $5.19(2.33)$ & $0.62,9.75$ & 0.026 \\
\hline Cough-MCLC & $3.38(1.87)$ & $-0.29,7.05$ & 0.07 & $2.50(2.41)$ & $2.22,7.23$ & 0.3 \\
\hline Fatigue-FACIT & $2.84(2.70)$ & $-2.45,8.13$ & 0.3 & $4.98(2.89)$ & $-0.68,10.63$ & 0.09 \\
\hline Lung Cancer Symptom Scale & $8.83(4.29)$ & $0.42,17.24$ & 0.04 & $6.12(4.40)$ & $-2.50,14.75$ & 0.16 \\
\hline \multicolumn{7}{|l|}{ HADS } \\
\hline Anxiety scale & $1.33(1.08)$ & $-0.80,3.46$ & 0.22 & $0.25(0.97)$ & $-1.66,2.15$ & 0.8 \\
\hline Depression scale & $0.99(1.10)$ & $-1.16,3.13$ & 0.37 & $0.59(1.19)$ & $-1.75,2.93$ & 0.62 \\
\hline EQ-5D-3L Score & $-0.75(0.07)$ & $-0.20,0.05$ & 0.25 & $-0.17(0.65)$ & $-0.30,-0.04$ & 0.009 \\
\hline EQ-5D-3L Health State Today (VAS) & $-10.21(5.47)$ & $-20.93,0.51$ & 0.05 & $-9.88(5.54)$ & $-20.75,0.99$ & 0.08 \\
\hline
\end{tabular}

NRS numerical rating scale, MCLC Manchester Cough in Lung Cancer Scale, HADS Hospital Anxiety and Depression Scale, EQ-5D Euro-Qual, VAS visual analogue scale, RDSI Respiratory Distress Symptom Intervention

to the intervention. Breathing exercises were practiced daily for the first 4 weeks between 87 and $100 \%$, and weekly practice for the remaining 8 weeks was between 96 and $100 \%$; acupressure was practiced for the first 4 weeks between 84 and $100 \%$, and weekly practice for the remaining 8 weeks was between 91 and $96 \%$; cough easing techniques were practiced daily for the first 4 weeks between 32 and $63 \%$, and weekly practice for the remaining 8 weeks was between 36 and $54 \%$. The lower use of cough easing techniques is likely to be related to the fewer number of participants that were bothered by cough in the intervention group (35/50).

The majority of participants reported that the breathing techniques were useful at least a little bit over the 12 weeks; there were only seven responses to not at all (five on day 1 and one during weeks 8 and 12). For cough easing techniques, there were six responses to not at all, all during the first 4 weeks post-intervention. Eighteen not at all responses were reported for usefulness of acupuncture; 16 were within the first 4 weeks.

\section{Discussion}

The aim of this study was to assess the feasibility and acceptability of a clinical trial of a new non-pharmacological intervention for the management of the Respiratory Distress Symptom Cluster, consisting of breathlessness-cough-fatigue, in patients with lung cancer. This study has demonstrated that it is feasible to conduct a larger trial that will recruit a sufficient number of patients to determine whether or not the intervention, RDSI, results in improved patient outcomes and is cost-effective, compared to the control group. Participants reported a high level of adherence to the different RDSI techniques, and the majority of participants reported that each component was useful over the 12 -week study period; both are important indicators of acceptability.

Screening identified that a proportion of patients were not eligible to participate due to current or recent treatment with chemotherapy or radiotherapy ( $21 \%$ ). Radiotherapy and chemotherapy can improve the symptoms of breathlessness and cough, but not fatigue [5], and hence may dilute the effect of the intervention or lead to unclear results. In addition, our previous work on intervention preferences demonstrated that many patients are reluctant to participate in a study which requires engagement of the sort needed to learn nonpharmacological techniques whilst having such treatments [17-19]. Therefore, we will retain these exclusion criteria in the main trial but will decrease the post-chemotherapy time from 4 weeks to 2 weeks and keep post radiotherapy at 4 weeks. The follow-on trial will have a built-in mechanism for re-contacting patients following the exclusion time period.

Screening for potential participants in this multisite feasibility study was challenging. Different sites often approached this aspect differently, and it was difficult to obtain accurate figures for non-participation in patients who met the inclusion 
criteria on initial screening of medical notes. Across the 11 recruitment sites, 128 patients fell into this category mostly due to the attending clinician not informing the patient about the study for reasons such as medical issues or further investigations required, personal/family issues, time constraints in a busy clinic and forgetting to mention the study during the consultation.

Eligible patients were required to report the presence of at least two of the three symptoms, in any combination, and perceive them to be bothersome. The combination of symptoms varies between and within patients and overtime [1]; however, we only collected this information as part of eligibility criteria. Feasibility data showed that, at study entry, breathlessness and fatigue were present and bothersome for more than $97 \%$ of participants, and cough was present for $80 \%$ and bothersome for $80 \%$ of those patients ( $66 \%$ of the total sample). It is possible that the approach used for screening questions resulted in fewer patients reporting bothersome cough, and this needs to be amended in a future trial. Nonetheless, these figures are representative of the lung cancer population for symptom prevalence [1-5]. Thirty percent of participants were postcurative treatment, and we expect that this number can increase through recruitment strategies targeting early diagnosis and survivors post-radical treatment. Therefore, a $20 \%$ attrition rate was used to determine sample size for the follow-on study and is comparable with similar studies in the past [10].

The method used for symptom screening consisted of two questions: 'Do you have breathlessness'; if 'yes', 'Are you bothered by your breathlessness?', and repeated for each symptom. This resulted in $25 \%$ of those screened not meeting inclusion criteria. Following the feasibility study, we consulted with a patient group not involved in the trial, who felt that asking whether or not a symptom was present was too simplistic and that the term bothered was suggestive of the patient 'complaining'. For the follow-on trial, we will adopt the single screening question suggested by the group: 'Are you affected in your day-to-day life by breathlessness?' and repeated for each symptom. Patients who at first approach report only one or no symptoms will be reassessed for their study eligibility at monthly intervals or until consent is withdrawn.

Despite the number of questionnaires that required completion, there was less than $5 \%$ missing data. The research team were conscious of the potential for responder burden during the trial. Training was provided to the research assistants and other trial recruiters to ensure that potential participants were fully-informed about the amount of required questionnaire completion. Follow-up packs were presented in colourcoded sections and presented in organised folders to help motivate people to complete them. Ethical approval was also sought to provide for one follow-up reminder telephone call. Few patients withdrew due to the amount of required paperwork. These techniques appeared to be successful and will be used in the subsequent trial.
This study was unique in that the intervention included a 'package' of different techniques that patients could choose from depending on their symptom experience at any particular time. Patients could use one or all of the techniques throughout the duration of the study. This is a similar approach successfully used in the management of breathlessness with complex interventions, whereby a number of different techniques are taught to patients $[9,10,20]$; however, we included interventions that also targeted cough and fatigue. In view of the evidence for structured activity for the management of cancerrelated fatigue $[39,40]$, the follow-on trial will include an individually tailored exercise plan as a core component of RDSI. This was also supported by our patient representative group. Likewise, Chan and colleagues [41] evaluated a nonpharmacological intervention for the management of anxietybreathlessness-fatigue in lung cancer; the intervention consisted of a combination of education and relaxation and was reported to improve patients' symptom experience.

Since the intervention was targeted at alleviating two or three distressing symptoms, part of our aim was to determine the most appropriate outcome measure for each symptom and to determine the MCID of the assessments used, allowing a sample size calculation for a powered study. The feasibility study included NRS scales in addition to the Dyspnoea-12, and we found that the Dyspnoea-12 provided the largest effect size, compared to any of the single item NRS scores. It also provides an assessment of total breathlessness severity that incorporates its physical discomfort and emotional consequences; this negates the need to use multiple NRS scores [21].

One challenge of evaluating the effectiveness of interventions aimed at ameliorating a cluster of symptoms is identification of a suitable outcome measure. The complexity of the Respiratory Distress Symptom cluster is an issue, whereby the combination of symptom occurrence differs between and within patients over time [1]. We applied a symptomspecific measure for each symptom in the cluster which necessitated a sample size calculation to be performed for each symptom. That analysis determined that a sample size of 122 patients per arm, based on the Dyspnoea-12 for breathlessness, would enable a meaningful difference to be captured in the outcome assessment used for cough and fatigue. Therefore, the follow-on study will have three primary outcomes: breathlessness, cough and fatigue. Consideration was also given to providing a composite score for the symptom cluster, as done in a past study [41]. However, this proved difficult since patients experienced symptoms in different combinations, with some experiencing all three symptoms and some experiencing only two. In this study, symptom prevalence was only collected as baseline.

This study was able to demonstrate the sensitivity of the EQ-5D-3 L to detect relevant changes in HRQoL. Recent developments by the EuroQol group have made available an updated version of the instrument to include five levels for 
each domain resulting in the EQ-5D-5 L, thereby providing a potentially more sensitive measure for use in the follow-on trial [42].

Due to the nature of the intervention and trial design, blinding was not possible for the therapist delivering the intervention. Researchers' collecting outcome data were not blind to allocation which possess an additional limitation. Researcher blinding to group allocation will be implemented for the collection of outcome measures in the follow-on study. The study was underpowered, focusing primarily on feasibility issues; hence, interpretation of comparative data between groups should be with caution. Data on oxygen use or steroids was not collected, and this may influence some of the results. Also, the groups differed in relation to COPD status and total symptom burden, two variables that need to be considered more carefully in a future trial and balanced accordingly.

\section{Conclusion}

The study has provided evidence of the feasibility and acceptability of a new intervention in the lung cancer population and warrants a fully powered trial before we reach any conclusions. Results were positive, with activity for RDSI at 4 and 12 weeks, a trend towards better results in the intervention group. Intervention acceptability was confirmed, and of particular note, no patients withdrew from the intervention group after week 4 which suggests that once participants became familiar with the intervention, they remained in the study. In this population of patients in often advanced stages of their illness and with complex symptomatology but limited symptom management options, it is imperative to test and, if found effective, subsequently to introduce new interventions that can improve the patients' symptom experience.

Acknowledgments This study was funded by a grant from Marie Curie Cancer Care (Ref: C16396/A14093). The authors thank the following dedicated research staff and advisors Peter Mackereth, Joanne Bayly, Loraine Morgan, Paula Maycock, Eileen Hackman, Claire Gregory, Chris Bailey and Ian Jacob. The authors are also grateful to all patients who took time to take part in this study.

Disclosures No conflict of interest for all authors and no disclosures to be reported.

Open Access This article is distributed under the terms of the Creative Commons Attribution-NonCommercial 4.0 International License (http:// creativecommons.org/licenses/by-nc/4.0/), which permits any noncommercial use, distribution, and reproduction in any medium, provided you give appropriate credit to the original author(s) and the source, provide a link to the Creative Commons license, and indicate if changes were made.

\section{References}

1. Molassiotis A, Lowe M, Blackhall F, Lorigan P (2011) A qualitative exploration of a respiratory distress symptom cluster in lung cancer: cough, breathlessness and fatigue. Lung Cancer 71(1):94-102

2. Cheville AL, Novotny PJ, Sloan JA, Basford JR, Wampfler JA, Garces YI, Jatoi A, Yang P (2011) Fatigue, dyspnea, and cough comprise a persistent symptom cluster up to five years after diagnosis with lung cancer. J Pain Symptom Manag 42(2):202-12

3. Edmonds P, Higginson I, Altmann D, Sen-Gupta G, McDonnell M (2000) Is the presence of dyspnea a risk factor for morbidity in cancer patients? J Pain Symptom Manag 19(1):15-22

4. Molassiotis A, Lowe M, Ellis J, Wagland R, Bailey C, LloydWilliams M, Tishelman C, Smith J (2011) The experience of cough in patients diagnosed with lung cancer. Support Care Cancer 19(12):1997-2004

5. Hofman M, Ryan JL, Figueroa-Mosele CD, Jean-Pierre P, Morrow GR (2007) Cancer related fatigue: the scale of the problem. Oncologist 12(Suppl 1):4-10

6. Dodd MJ, Miaskowski C, Paul SM (2001) Symptom clusters and their effect on the functional status of patients with cancer. Oncol Nurs Forum 28(3):465-470

7. Molassiotis A, Uyterlinde W, Hollen PJ, Sarna L, Palmer P, Krishnasamy M (2015) Supportive care in lung cancer: milestones over the past 40 years. J Thorac Oncol 10(1):10-18

8. Yorke J, Brettle A, Molassiotis A (2012) 12. Nonpharmacological interventions for managing respiratory symptoms in lung cancer. Chron Respir Dis 9(2):117-29

9. Bausewein C, Booth S, Gysels M, Higginson I (2008) Nonpharmacological interventions for breathlessness in advances stages of malignant and non-malignant disease. Cochrane Database Syst Rev 2, CD005623

10. Farquhar MC, Prevost AB, McCrone P, Brafman-Price B, Bentley A, Higginson IJ, Todd C, Booth S (2014) Is a specialist breathlessness service more effective and cost effective for patients with advanced cancer and their carers than standard care? Findings of a mixed-method randomised controlled trial. BMC Med 12:194

11. Zick SM, Alrawi S, Merel G, Burris B, Sen A, Litzinger A, Harris RE (2011) Relaxation acupressure reduces persistent cancer-related fatigue. Evidence-based Complemen Altern Med; Article ID 142913, 10 pages

12. Molassiotis A, Sylt P, Diggins H (2007) The management of cancer-related fatigue after chemotherapy with acupuncture and acupressure: a randomized controlled trial. Complement Ther Med 15(4):228-237

13. Vickers AJ, Straus DJ, Fearon B, Cassileth BR (2004) Acupuncture for post chemotherapy fatigue: a phase II study. J Clin Oncol 22(9): 1731-1735

14. Chamberlain S, Birring SS, Garrod R (2014) Non-pharmacological interventions for refractory chronic cough patients: systematic review. Lung 192:75-85

15. Vertigan AE, Theodoros DG, Gibson PG, Winkworth AL (2006) Efficacy of speech pathology management for chronic cough: a randomised placebo controlled. Thorax 61:1065-69

16. Medical Research Council (2000) A framework for development and evaluation of RCTSs for complex interventions to improve health. London

17. Ellis J, Wagland R, Tishelman C, Williams ML, Bailey CD, Haines J, Caress AC, Lorigan P, Smith JA, Booton R, Blackhall F, Molassiotis A (2012) Considerations in developing and delivering a non-pharmacological intervention for symptom management in lung cancer: the views of patients and informal caregivers. J Pain Symptom Manag 44(6):831-42

18. Wagland R, Ellis J, Bailey CD, Haines J, Caress A, Williams ML, Lorigan P, Smith J, Tishelman C, Booton R, Luker K, Blackhall F, 
Molassiotis A (2012) Considerations in developing and delivering a non-pharmacological intervention for symptom management in lung cancer: the views of health care professionals. Support Care Cancer 20(10):2565-74

19. Molassiotis A, Emsley R, Ashcroft D, Caress AC, Ellis J, Wagland R, Bailey CD, Haines J, Williams ML, Lorigan P, Smith J, Tishelman C, Blackhall F (2012) Applying Best-Worst scaling methodology to establish delivery preferences of a symptom supportive care intervention in patients with lung cancer. Lung Cancer 77(1):199-204

20. Barton R, English A, Nabb S, Rigby AS, Johnson MJ (2010) A randomised trial of high vs low intensity training in breathing techniques for breathless patients with malignant lung disease: a feasibility study. Lung Cancer 70(3):313-9

21. Yorke J, Moosavi SH, Shuldham C, Jones PW (2010) Quantification of dyspnoea using descriptors: development and initial testing of the Dyspnoea-12. Thorax 65(1):21-6

22. Yorke J, Armstrong I (2013) Assessment of breathlessness in pulmonary hypertension: validation of the Dyspnoea-12. Eur J Cardiovasc Nurs 13(6):506-14

23. Yorke J, Swigris J, Russell AM, Kwong G, Jones PW (2013) Dyspnoea-12 is a valid and reliable measure of breathlessness in patients with interstitial lung disease. Chest 139(1):159-164

24. Molassiotis A, Ellis J, Wagland R, Lloyd Williams M, Blackhall F, Yorke J, Smith JE (2012) The Manchester Cough in Lung Cancer scale: the development and validation of a new assessment tool. $\mathrm{J}$ Pain Symptom Manag 45(2):179-90

25. Yellen SB, Cella DF, Webster K, Blendowski C, Kaplan E (1997) Measuring fatigue and other anemia-related symptoms with the Functional Assessment of Cancer Therapy (FACT) measurement system. J Pain Symptom Manag 13(2):63-74

26. Hollen PJ, Gralla RJ, Kris MG, Cox C, Belani CP, Grunberg SM, Crawford J, Neidhart JA (1994) Measurement of quality of life in patients with lung cancer in multicenter trials of new therapies: psychometric assessment of the Lung Cancer Symptom Scale. Cancer 73:2087-2098

27. EuroQoL Group (1990) EuroQoL: a new facility for the measurement of health-related quality of life. Health Policy 16:199-208

28. Kind P, Hardman G, Macran S (1999) UK population norms for EQ-5D. York Centre for Health Economics, Discussion Paper, University of York
29. Zigmond AS, Snaith PR (1983) The hospital anxiety and depression scale. Acta Psychiatr Scand 67:361-370

30. Lancaster GA, Dodd S, Williamson PR (2004) Design and analysis of pilot studies: recommendations for good practice. J Eval Clin Pract 10(2):307-12

31. Liang KY, Zeger SL (1986) Longitudinal data analysis using generalized linear models. Biometrika 73:13(22)

32. Twisk JWR (2003) Applied longitudinal data analysis for epidemiology: a practical guide. Cambridge University Press

33. Oxberry SG, Bland MJ, Clark AL, Cleland JGF, Johnson M (2012) Minimally clinically important difference in chronic breathlessness: every little helps. Am Heart J 164(2):229-35

34. de Kleijn WP, De Vries J, Wijnen PA, Drent M (2011) Minimal (clinically) important differences for the Fatigue Assessment Scale in sarcoidosis. Respir Med 105:1388-1395

35. Johnson MJ, Bland JM, Oxberry SG, Abernethy AP, Currow DC (2013) Clinically important differences in the intensity of chronic refractory breathlessness. J Pain Symptom Manag 46(6):957-63

36. Pouchot J, Kherani RB, Brant R, Lacaille D et al (2008) Determination of the minimally important difference for seven fatigue scales in rheumatoid arthritis. J Clin Epidemiol 61(7):705713

37. Borm GF, Fransen J, Lemmens WA (2007) A simple sample size formula for analysis of covariance in randomised clinical trials. J Clin Epidemiol 60(12):1234-1238

38. Yost KJ, Eton DT (2005) Combining distribution and anchor-based approaches to determine minimal clinical differences: the FACIT experience. Eval Health Prof 28(2):172-191

39. Speck RM, Courneya KS, Masse LC, Duval S, Schmitz KH (2012) An update on controlled physical activity trials in cancer survivors: a systematic review and meta-analysis. J Cancer Surviv 4:87-100

40. Schwartz AL, Mori M, Gao R, Nail LM, King M (2001) Exercise reduces daily fatigue in women with breast cancer receiving chemotherapy. Med Sci Sports Exerc 33(5):718-723

41. Chan CWH, Richardson A, Richardson J (2011) Managing symptoms in patients with advanced lung cancer during radiotherapy: results of a psychoeducational randomised controlled trial. J Pain Symptom Manag 41(2):346-348

42. Brazier J, Ratcliffe J, Salomon JA, Tsuchiya A (2007) Measuring and valuing health benefits for economic evaluation. Oxford University Press, Oxford 